\title{
Analysis and Comparison of the Tongue Picture of 34 Patients with Severe Acute Respiratory Syndrome*
}

\author{
WU Hong-jin (吴红金 $)^{1}$, WANG Fan(王 凡 $)^{1}$, WANG Bin (王 斌) ${ }^{1}$, WANG Sheng-qi (王升启 $)^{2}$, \\ ZHAO Xi-yin (赵锡银 $)^{1}$, YAO Jun (姚 军) ${ }^{1}$, XU Ping (许 萍 $)^{1}$, and DUAN Shu-wei (段姝伟) ${ }^{1}$
}

\begin{abstract}
Objective: To explore the changes of tongue pictures in the developing process of disease in patients with severe acute respiratory syndrome (SARS), and to compare the tongue pictures of SARS patients with those of suspected SARS patients. Methods: Materials of tongue picture were dynamically collected from 34 SARS patients and 35 suspected SARS patients, and the difference and similarity between them were analyzed. Meanwhile, the changes in clinic symptoms and tongue picture after integrative medicinal treatment were observed. Results: Characteristics of tongue picture in SARS patients were changed along with the progress of the disease, showing that there existed a tendency of the pathogen invading from exterior to interior, from surface to the deep. Also the tongue pictures were varied due to the coexistence of dampness pathogen; the time of the early stage was generally $1-1.5$ days, shorter than that of other stages. While in the patients with suspected SARS, the tongue picture was mostly red with thin white or yellow coating, comparatively steady and showing no obvious change along with the development of the disease. Conclusion: Observing the tongue picture and its changes in different stages can be helpful to understanding the property of pathogenic evil and its developing rules, thus offering a basis for clinical treatment.

KEY WORDS severe acute respiratory syndrome, suspected SARS, tongue picture
\end{abstract}

Tongue picture is an important part in the diagnostics of traditional Chinese medicine (TCM), which is especially indispensable in Syndrome differentiation of epidemic febrile disease. Its importance also counts a lot in enhancing the accuracy in diagnosis and treatment of severe acute respiratory syndrome (SARS), a disease which belongs to the epidemic febrile category of TCM. This prospective survey was planned by the authors for observing and analyzing the change of tongue pictures in 34 SARS patients hospitalized in Beijing Municipal Hospital of Traditional Chinese and Western Medicine (ICWM), one of the appointed SARS hospital in Haidian District of Beijing . The tongue picture of 35 patients with suspected SARS was also observed and analyzed for comparison. The results were reported as follows.

\section{METHODS}

\section{Clinical Materials}

All the 34 SARS patients enrolled had their diagnosis conforming to "Clinical Diagnostic Standard of Infective Atypical Pneumonia (Trial)" promulgated by Ministry of Health ${ }^{(1)}$. And the 35 patients, who were diagnosed as suspected SARS at the first visit but then were excluded from SARS later, were also enrolled for control. Among them, 27 were finally diagnosed as suffering from pulmonary infection, 4 from upper respiratory tract infection, 2 from pulmonary tuberculosis complicated with infection, 1 from chronic bronchitis with infection and 1 from diabetes mellitus with pulmonary infection.

Among the 34 SARS patients, 17 were males and 17 females, their ages ranging from 15 to 83 years, with the average age of $33.15 \pm 16.03$ years. Seven of them were younger than 20 years old, 13 between 21 and 30,6 between 31 and 40,5 between 41 and 50 and 3 over 50 years old. Their disease course was $0-20$ days, averaging $4.77 \pm$ 3.87 days, with 22 of them between 0 and 4 days, 9 between 5 and 10 days and 3 above 10 days.

As for the 35 patients with suspected SARS,

\footnotetext{
* Key project No. 863 of the Tenth Five-Year Plan, Ministry of Sciences and Technology, Study on Important Technique and Product Development for SARS Prevention and Treatment (2003AA208101);

Project of State Administration of Traditional Chinese Medicine ( special subject for SARS No.06)

1. Beijing Municipal Hospital of Integrated Traditional Chinese and Western Medicine, Beijing (100039); 2. Academy of Military Medical Sciences

Correspondence to: Dr. WU Hong-jin

Tel:010-68179234;E-mail:dshuw@sohu.com
} 
they were 24 males and 11 females, ages ranging from $15-73$ years old, averaging $35.06 \pm 18.81 .7$ of them were younger than 20 years, 13 between $21-30$ years, 5 between $31-40,5$ between $41-$ 50 and 5 over 50 ; their course of disease was $0-30$ days, averaging $4.86 \pm 6.41$ days, with 24 between $0-4$ days, 7 between $5-10$ and 4 above 10 days.

There was no statistical difference (analyzed by Chi-square test) in sex, age and course of disease between the two groups, and therefore they were comparable.

\section{Therapeutic Method}

\section{Treatment for SARS patients}

All the SARS patients were treated with basic Western medicine according to the "Therapeutic Scheme for Infective SARS" promulgated by the Ministry of Health, P.R. China ${ }^{(2)}$ in the following way: (1) Anti-virus drugs: $500 \mathrm{mg}$ Ribaviria was given through intravenous dripping, once or twice daily for 5-14 days. (2) Anti-infection drugs: for patients with infective complication, Levofloxacin $200 \mathrm{mg}$ was given by intravenous dripping or oral intake once or twice daily, or $500 \mathrm{mg}$ Azithromycin given by intravenous dripping once or twice daily, or cephalosporin used. (3) Immunity enhancing agents: Thymus peptide $200 \mathrm{mg}$ was chosen and given through intravenous dripping once daily. (4) Glucocorticoid was given to serious patients or patients with serious toxic symptoms such as continuous high fever or having patchy shadows shown in chest X-ray film. The medication was scheduled according to their condition: methylprednisolone $40-80 \mathrm{mg}$ was given by intravenous dripping once or twice daily at the beginning. The maximum dosage could be $320 \mathrm{mg}$ per day according to the disease condition. After the patients' body temperature got normalized for over 3 days, and chest film showed absorption and improvement in pulmonary inflammation, the dose of methylprednisolone would be reduced by $40-120 \mathrm{mg}$ every $2-3$ days, until it was reduced to less than $40 \mathrm{mg}$ per day given by intravenous dripping once daily or oral intake in two times. Then the medication was finally stopped $2-3$ days later. Close observation of body temperature should be done during the process of reducing dosage, to stop the reduction or even to go back to the last dosage was necessary when temperature fluctuated.

TCM treatment was given additionally to 29 SARS patients by giving Houttuyniae injection of $100 \mathrm{ml}$ (product of Sanjiu Ya' an Pharmaceutical
Factory, Batch No. 030406) to those with body temperature under $38.5^{\circ} \mathrm{C}$ by intravenous dripping once daily; or Qingkailing injection of $20-40 \mathrm{ml}$ (product of the Pharmaceutic Factory of Beijing University of Chinese Medicine, with its major components as cholalic acid, pearl shell, hyodeoxy cholic necleic acid, cape jasmine fruit, buffalo horn, indigowood root, baicalin and honeysuckle flower) to those with body temperature above $38.5^{\circ} \mathrm{C}$ by intravenous dripping once or twice daily.

For patients who still felt chest distress, shortness of breath, cold limbs or slow heart rate, in spite of their body temperature already lowered to normal range, $40 \mathrm{ml}$ of Shengmai injection (each milliliter containing $0.1 \mathrm{~g}$ of red ginseng, $0.312 \mathrm{~g}$ of dwarf lilyturf root and $0.156 \mathrm{~g}$ of Chinese magnoliavine fruit, product of Sanjiu Ya' an Pharmaceutic Factory, Batch No. 021203) was given by intravenous dripping once daily.

Different TCM decocted recipes were given according to conditions of disease and clinical symptoms. For patients in the early stage ( $1-5$ days after onset), in whom the pathogenesis was chiefly blockage of Damp-heat involving both Wei-fen (卫 分) and Qi-fen (气分), Modified Yinqiao powder and Sanren decoction (银剋散合三仁汤加减) were given, which consisted of honeysuckle flower $15 \mathrm{~g}$, forsythia fruit $15 \mathrm{~g}$, almond $12 \mathrm{~g}$, talcum $15 \mathrm{~g}$, white amonum fruit $5 \mathrm{~g}$ (smashed, decocted later), officinal magnolia bark $6 \mathrm{~g}$, Pinellia ternata $10 \mathrm{~g}$, coix seed $20 \mathrm{~g}$, batryticated silkworm $6 \mathrm{~g}$, Tumeric $9 \mathrm{~g}$, coat of cicada $6 \mathrm{~g}$, Chinese atractylodes $6 \mathrm{~g}$, sweet worm herb $10 \mathrm{~g}$ (decocted after other herbs) and baikal skullcap root $10 \mathrm{~g}$. For patients in the middle stage (about $3-10$ days after onset), in whom the pathogenesis was mainly noxious dampheat accumulation to block Qi flow, Modified Ganlu Xiaodu Pill (甘露消毒丹加减) was given, which consisted of plaster $30 \mathrm{~g}$ (decocted before other herbs), fried Almond $10 \mathrm{~g}$, white amonum fruit $6 \mathrm{~g}$ ( smashed, decocted after decoction of other herbs), talcum $20 \mathrm{~g}$, mulberry silkworm $10 \mathrm{~g}$, coat of cicada $6 \mathrm{~g}$, Chinese atractylodes $6 \mathrm{~g}$, tumeric 10 $\mathrm{g}$, rhizoma acori tatarinowii $10 \mathrm{~g}$, pinellia ternata 10 $\mathrm{g}$, Chinese thorowax root $12 \mathrm{~g}$, baikal skullcap root $10 \mathrm{~g}$, red root gromwell root $15 \mathrm{~g}$, sweet wormweed herb $10 \mathrm{~g}$, mulberry bark $10 \mathrm{~g}$ and Tansy Mustard seed $10 \mathrm{~g}$. For patients in the ultimate stage (mostly 7-14 days after onset), in whom the pathogenesis was chiefly toxic Damp-heat exuberance and Qi exhaustion, with inner occlusion of blood stasis, Modified Qingying Decoction (清营 汤) combined with Shengmai Powder (生脉散) 
was given, which consisted of buffalo horn $30 \mathrm{~g}$, rehmannia dried rhizomae $15 \mathrm{~g}$, kakuda figwort root $15 \mathrm{~g}$, honeysuckle flower $15 \mathrm{~g}$, American ginseng $5 \mathrm{~g}$ (braized separately), dwarf lilyturf root 10 $\mathrm{g}$ and pulp of dogwood fruit $15 \mathrm{~g}$. The recipes were given one dosage per day by immersing the drugs in water for $15-30$ min firstly, and then decocted in routine manner to $200 \mathrm{ml}$ and taken in two or three portions according to the conditions of the patients.

\section{SARS}

2. Treatment of patients with suspected

These patients were treated with the same method as that for SARS patients except that glucocorticoid was not given. The TCM recipe given to 33 patients of them was that formulated specially for suspected SARS patients worked out by specialists at the request of Beijing Bureau of TCM with the following components: honeysuckle flower $15 \mathrm{~g}$, forsythia fruit $15 \mathrm{~g}$, heartleaf houttuynia herb $25 \mathrm{~g}$, great burdock achene $10 \mathrm{~g}$, Almond $10 \mathrm{~g}$, common hogfennel root $10 \mathrm{~g}$, lobed kudzurine root $15 \mathrm{~g}$, peppermint $10 \mathrm{~g}$ (to be decocted later than others), gypsum $30 \mathrm{~g}$ (decocted earlier than others), Talcum $10 \mathrm{~g}$ (wrapped), liquorice root $6 \mathrm{~g}$, common reed rhizome $15 \mathrm{~g}$. The recipe may be modified according to conditions: mulbarry bark, flower of loquat, cicada skin and tendril-leaved fritillary bulb would be added for patients with severe cough; baikal skullcap root, Chinese paris rhizome, common anemarrhena rhizome and calcite added for patients with high fever; fobes notopterygium rhizome, honeysuckle stem, coix seed and Chinese atractylodes added for patients with headache and general aching; dwarf lilyturf root, coastal glehnia root and dendrobium stem added for pa- $^{-}$ tients with dry mouth and severe thirst; rhubarb and giant knotweed added for patients with dry stool; agastache rugosus, fortune eupatorium herb, malt, hawthorn fruit and medicated leaven added for patients with greasy coating, chest stiffness, and anorexia; pseudostellaria root and milkvetch (astragalus) root added for patients with lassitude; Chinese thorowax root, bitter orange, nutgrass galingale rhizome and bamboo shavings added for patients with depressed spirit; powder of Antelope, amber, nacre, uncaria added for manic patients. The medicinal herbs were decocted routinely and taken in two portions in the morning and evening, one dose every day, or, for severe patients, 3 doses in 2 days, taken in three portions every day in the morning, noon and evening. Six days of treatment was regarded as a therapeutic course. Decoction could be given continuously ac- cording to individual conditions.

\section{Items and Methods of Observation}

\section{Tongue picture}

Photo of tongue picture was taken every morning at 8:00-10:00 under natural light, with Kodak Easy Share LS443 digital camera (4 million picture elements) in close-up view within $15 \mathrm{~cm}$. Then the photos were transferred to the information collecting room to make the pictures consistent by regulating the light to meet the requirement of comparison. Analysis was conducted coordinately by two senior doctors with over 20 years of experience in the field. However, since there was no special clinic for patients with fever and the patients of SARS had no choice but to be collected to a certain hospital, abscondence was unavoidable.

\section{Clinic symptom improving time}

The improving time of 10 primary clinic symptoms, including fever, head distending pain, general aching, cough, short breath, poor appetite, dry mouth and thirst, fatigue, sweating and palpitation were recorded.

3. Test of coronavirus nucleic acid and its anti-body

Coronavirus nucleic acid (CNA) in serum and/or acquired mouth-rinsing liquid of 21 SARS patients and 26 suspected SARS patients were detected using RT-PCR fluorometry with Cycler IQ Multi-Color Real Time PCR Detection System (product of BioRad Co. USA). And the anti-body (Anti-CNA) was detected in the meantime by ELISA using enzyme linked immunosorbent analyser (Labsystems Dragon, Finland) with SARS Coronavirus Serum Anti-body Test Kit (batch No. 20030525) provided by Institute of Radiation Medicine, Academy of Military Medical Sciences.

\section{Statistical Analysis}

Chi-square $\left(\chi^{2}\right)$ test and $t$-test were adopted.

\section{RESULTS}

\section{Changes of Tongue Pictures in SARS Patients at Different Stages}

Shown in Table 1 and Fig 1 (See inside back cover). The tongue picture in most SARS patients ( $16 / 28$, accounting for $57.1 \%$ ) in the early stage proved to be red with thin white or yellow coating; in the middle stage, it was mostly ( $17 / 32$, accounting for $53.1 \%$ ) red with white or thick 
Table 1. Disease Course and Change of Tongue Pictures in 34 SARS Patients (Cases)

\begin{tabular}{|c|c|c|c|c|c|}
\hline \multicolumn{2}{|c|}{ Tongue Picture } & \multicolumn{4}{|c|}{ Stage } \\
\hline Proper & Coating & Early & Middle & Ultimate & Recovery \\
\hline Red & $\begin{array}{l}\text { thin white } \\
\text { or yellow }\end{array}$ & 16 & 1 & 1 & 6 \\
\hline Red & $\begin{array}{l}\text { white or } \\
\text { thick yellow }\end{array}$ & 4 & 17 & 6 & 7 \\
\hline Red & greasy white & 4 & 5 & 8 & 2 \\
\hline Red & $\begin{array}{l}\text { greasy } \\
\text { yellow }\end{array}$ & 3 & 8 & 9 & 2 \\
\hline Crimson & little dry & 1 & 1 & 4 & 10 \\
\hline Total & & 28 & 32 & 28 & 27 \\
\hline
\end{tabular}

yellow coating; in the ultimate stage, the tongue coatings were varied, but mainly thick and greasy; and in the recovery stage, it was mostly $(10 / 27,37.0 \%)$ red and dry with little coating.

\section{Tongue Pictures in Suspected SARS Patients at Different Stages}

Shown in Table 2 and Fig 2 (See inside back cover). The tongue picture of suspected SARS patients, no matter in what stage of the disease the patient was, was primarily red with thin white or yellow fur in most cases, accounting for $72.7 \%(16 / 22)$ in the early stage, $53.8 \%(14 / 26)$ in the middle stage, $60 \%(18 / 30)$ in the ultimate stage, and $54.8 \%(17 / 31)$ in the recovery stage.

\section{Clinical Symptom Improving Time}

Clinical symptom improving time was counted in 30 out of the 34 SARS patients, whose hospitalization time was more than 5 days. Result showed that the average improving time of various symptoms was $5-7$ days. Tongue picture was basically improved in synchronization with the improvement of other symptoms.

\section{Changes of Tongue Picture of SARS Patients in Hospitalization Period}

Changes of tongue picture were sorted into three types: (1) Improved: tongue color changed from crimson to red or from red to slight red, and tongue coating changed from thick (greasy) to thin or from yellow to white. (2) Unchanged: No change occurred in tongue color or coating. (3) Aggravated: the changes of tongue picture went contrary to that of the improved.

Table 2. Disease Course and Change of Tongue Pictures in 35 Suspected SARS Patients (Cases)

\begin{tabular}{|c|c|c|c|c|}
\hline \multirow{2}{*}{ Tougue picture } & \multicolumn{4}{|c|}{ Stage } \\
\hline & Early & Middle & Ultimate & Recovery \\
\hline $\begin{array}{l}\text { Red tongue with thin white } \\
\text { or thin yellow coating }\end{array}$ & 16 & 14 & 18 & 17 \\
\hline $\begin{array}{l}\text { Red tongue with white or } \\
\text { thick yellow coating }\end{array}$ & 5 & 7 & 6 & 5 \\
\hline $\begin{array}{l}\text { Red tongue with greasy } \\
\text { white coating }\end{array}$ & 0 & 1 & 3 & 3 \\
\hline $\begin{array}{l}\text { Red tongue with greasy } \\
\text { yellow coating (dirty) }\end{array}$ & 0 & 1 & 2 & 4 \\
\hline $\begin{array}{l}\text { Red tongue (crimson) with } \\
\text { little coating (little fluid) }\end{array}$ & 1 & 3 & 1 & 2 \\
\hline Total & 22 & 26 & 30 & 31 \\
\hline
\end{tabular}


Observation was performed on $21 \mathrm{pa}^{-}$ tients with hospitalization time over 8 days. Results showed that the tongue picture was improved in 16 patients, among them, 11 got improved 3 days after treatment, 3 within 6 days and 2 over 6 days after it. As to the other 5 patients, tongue picture was unchanged in 3 and aggravated in 2. See Fig 3 (See inside back cover).

\section{Positive Rate of CNA and Anti-CNA}

In the 21 SARS patients tested, serum positive CNA was found in 9 , positive antiCNA in 2, and both positive CNA and antiCNA in 1 . Besides, positive CNA was found in the mouth-rinsing liquid of 5 patients. The total positive rate was $80.95 \%(17 / 21)$. And in the 25 suspected SARS patients, only 2 showed positive serum CNA on the 4 th and 18 th day respectively after onset.

\section{DISCUSSION}

It could be seen from the comparison between the tongue pictures of 34 SARS patients and 35 suspected SARS patients that though the pathogens of diseases were both of an evil-warm nature and belonged to the same kind in TCM, the pathogenesis of them was different, therefore the influence of the pathogens on the human body is different. The tongue picture in SARS patients, which changed along with the development of SARS, showed that the process of SARS went from exterior to interior, and from surface to the inside. Because of the coexistence of dampness pathogen, the manifestations of tongue coating are varied. For example, red tongue with greasy white coating or greasy yellow coating (dirty fur) is commonly seen in the ultimate stage. Suspected SARS also belongs to epidemic febrile kind of disease, but because its pathogen is of common windwarm nature, no obvious changes in tongue picture occurred, and what was shown was mainly red tongue with thin white or thin yellow coating in the whole developing process of the disease. So, the authors held that the observation of the manifestations and changes of tongue picture could be helpful to understanding the property and developing rules of disease, which provides a reliable basis for clinical treatment.

SARS is quite a new disease for human beings. Although a great deal of basical and clinical studies have been conducted at home and abroad, the knowledge about SARS is still superficial. Since at present no special effective treatment for SARS is discovered in Western medicine, it is quite important to control the epidemic disease by actively using TCM and fulfilling the integration of TCM with Western medicine. Tongue picture is a very important part in TCM diagnostics, especially in the SARS ward, where medical staff has to closely defend themselves by as much physical separation of themselves from the patients as possible, it makes the feeling of pulse very difficult. So careful analysis of tongue picture becomes more important in confirming TCM Syndrome, revealing the developing rules of the disease, and promoting diagnosis and therapeutic level of ICWM.

Besides, it is found that the time of the early stage in the whole course of SARS, generally $1-1.5$ days, was shorter than that of other stages.

Since SARS was strongly infective, diagnosis for the confirmation of SARS and the exclusion of the suspected patients should be done very carefully. We hold that detailed epidemic investigation, careful analysis of clinical symptoms and TCM Syndrome, and corresponding combination of laboratory examinations could be helpful in improving the clinical level of ICWM in treating SARS.

\section{REFERENCES}

1. Ministry of Health, P. R. China. The clinical diagnostic criteria of SARS (Trial) (May 3, 2003).

2. Ministry of Health, P.R. China. The recommended treatment regimen of SARS(May 3, 2003).

(Received September 22, 2003) 